Research, Society and Development, v. 9, n. 10, e2789108381, 2020

(CC BY 4.0) | ISSN 2525-3409 | DOI: http://dx.doi.org/10.33448/rsd-v9i10.8381

\title{
Auditoria interna para a estratificação do problema da qualidade das equipes de enfermagem na COVID-19
}

Internal audit for the stratification of the quality problem of nursing teams at COVID-19

\section{Auditoría interna para la estratificación del problema de calidad de los equipos de enfermería en COVID-19}

Recebido: 11/09/2020 | Revisado: 17/09/2020 | Aceito: 24/09/2020 | Publicado: 26/09/2020

\author{
Alexandra de Oliveira Matias Ferreira \\ ORCID: https://orcid.org/0000-0003-1003-2754 \\ Universidade Federal Fluminense, Brasil \\ E-mail: alexandrauff@gmail.com \\ Zenith Rosa Silvino \\ ORCID: https://orcid.org/0000-0002-2848-9747 \\ Universidade Federal Fluminense, Brasil \\ E-mail: zenithrosa@id.uff.b
}

\section{Resumo}

Objetivo: descrever o gerenciamento da rotina do trabalho do dia a dia para análise da qualidade das equipes de enfermagem, por meio de uma auditoria interna, para determinar as não conformidades no cuidado ao doente grave com a COVID-19. Método: trata-se de um relato de experiência da aplicação do modelo de gerenciamento da rotina do trabalho do dia a dia, na auditoria interna da UTI de um hospital universitário, no Rio de Janeiro. O estudo contou com quatro fases, a coleta de dados foi realizada por meio de entrevista com formulário, por uma enfermeira especialista no modelo de gestão da qualidade total japonês. Foi empregado o ciclo de Plan, Do, Check e Act (PCDA), a análise descritiva e a análise de Pareto. Resultados: mesmo após a supervisão das equipes interprofissionais de enfermagem pelas coordenadoras da assistência de enfermagem, 94\% das equipes não modificaram seu comportamento e estavam em não conformidades, avaliadas pelas evoluções de enfermagem, aprazamento das prescrições médicas, roteiro de passagem de plantão e notificações de eventos adversos sensíveis à assistência de enfermagem. Considerações finais: apesar do controle de qualidade da assistência de enfermagem pelas enfermeiras gerentes ser efetivo, a auditoria interna mostrou que, direta ou indiretamente, as anomalias tinham relação com: a supervisão direta de enfermagem com uso ininterrupto de Equipamento de Proteção 
Individual (EPI); uso de estratégias de comunicação inefetivas; falta de rigor no processo de avaliação da admissão de novos profissionais de enfermagem; procedimentos operacionais padrões em desconformidade e a péssima capacitação realizada na admissão das equipes interprofissionais para atuar no tratamento da COVID-19 na UTI.

Palavras-chave: Auditoria de enfermagem; COVID-19; Admissão e escalonamento de pessoal; Cuidados críticos; Pesquisa em administração de enfermagem.

\section{Abstract}

Objective: to describe the management of the routine of day-to-day work for analysis of the quality of nursing teams, through an internal audit, to determine the non-conformities in the care of the severely ill patient with COVID-19. Method: this is an experience report of the application of the routine routine model of day-to-day work in the internal audit of the ICU of a university hospital in Rio de Janeiro. The study had four phases, data collection was performed through interview with form, by a nurse specialized in the japanese total quality management model. The Plan, Do, Check and Act (PCDA) cycle, descriptive analysis and Pareto analysis were used. Results: even after the supervision of the interprofessional nursing teams by the nursing care coordinators, $94 \%$ of the teams did not modify their behavior and were in non-conformities, evaluated by nursing evolutions, compliance with medical prescriptions, script of shift and notifications of adverse events sensitive to nursing care. Final considerations: although the quality control of nursing care by the nurse managers is effective, the internal audit showed that, directly or indirectly, the anomalies were related to: direct supervision of nursing with uninterrupted use of Personal Protective Equipment (PPE); use of ineffective communication strategies; lack of rigor in the process of evaluating the admission of new nursing professionals; standard operating procedures in non-conformity and poor training performed in the admission of interprofessional teams to act in the treatment of COVID-19 in the ICU.

Keywords: Nursing Audit; COVID-19; Personnel staffing and scheduling; Critical care; Nursing administration research.

\section{Resumen}

Objetivo: describir la gestión de la rutina del trabajo diario para el análisis de la calidad de los equipos de enfermería, a través de una auditoría interna, para determinar las inconformidades en la atención del paciente gravemente enfermo con COVID-19. Método: este es un informe 
de experiencia de la aplicación del modelo rutinario rutinario del trabajo diario en la auditoría interna de la UCI de un hospital universitario de Río de Janeiro. El estudio tuvo cuatro fases, la recopilación de datos se realizó a través de la entrevista con la forma, por una enfermera especializada en el modelo de gestión de calidad total japonés. Se utilizó el ciclo Plan, Do, Check and Act (PCDA), el análisis descriptivo y el análisis de Pareto. Resultados: incluso después de la supervisión de los equipos de enfermería interprofesional por parte de los coordinadores de enfermería, el 94\% de los equipos no modificó su comportamiento y se encontraban en inconformidades, evaluados por las evoluciones de enfermería, el cumplimiento de las prescripciones médicas, el guión de turno y las notificaciones de eventos adversos sensibles a la atención de enfermería. Consideraciones finales: aunque el control de calidad de la atención de enfermería por parte de los enfermeros es eficaz, la auditoría interna demostró que, directa o indirectamente, las anomalías estaban relacionadas con: supervisión directa de la lactancia con el uso ininterrumpido de equipos de protección personal (EPP); el uso de estrategias de comunicación ineficaces; falta de rigor en el proceso de evaluación de la admisión de nuevos profesionales de enfermería; procedimientos operativos estándar en materia de inconformidad y poca formación realizados en la admisión de equipos interprofesionales para actuar en el tratamiento del COVID-19 en la UCI.

Palabras clave: Auditoría de Enfermería; COVID-19; Cuidado crítico; Admisión y programación de personal; Investigación en administración de Enfermería.

\section{Introdução}

O hospital, como um sistema prestador de serviços, tem os seus serviços voltados a satisfazer as necessidades dos clientes. Porém, sabe-se que a vida das pessoas depende intimamente das empresas de saúde e estas dependem do trabalho de qualidade da equipe interprofissional de enfermagem (enfermeiros, técnicos e auxiliares de enfermagem).

$\mathrm{Na}$ prática, muitas vezes é dillcil separar habilidades, conhecimento e valor que capacitam um profissional para exercer sua função, mas as equipes são grupos de pessoas que devem interagir entre si para que objetivos comuns sejam alcançados (Mosser, G., \& Begun, 2015; Trovó, Cucolo., \& Perroca, 2020). Uma equipe interprofissional atua em prol de se alcançar as metas das empresas públicas ou empresas privadas de saúde. Entretanto, é possível destacar que pode existir um rol de desconformidades na prática da assistência de enfermagem, sendo que os auditores na enfermagem atuam em empresas (públicas ou 
privadas) com o intuito de detectar os problemas e mostrar os caminhos para as correções devidas.

Com a saída de antigos profissionais de enfermagem por fatores de riscos associados ao adoecimento pela forma grave da COVID-19, além dos demais que já apresentavam comorbidades em licença médica, a equipe interprofissional de enfermagem de muitos hospitais recebeu novos colaboradores de forma emergencial (Figueiredo, 2020). As equipes transitórias precisaram aprender a trabalhar em meio ao medo com novas rotinas do trabalho do dia a dia, mantendo-se em defesa aos eventos adversos aos pacientes, com Equipamento de Proteção Individual (EPI), tornando-se grandes desafios, principalmente para aqueles em faixas etárias menores.

Na enfermagem, como nas demais áreas profissionais da saúde, a grande maioria dos seus profissionais conciliam o trabalho com a formação profissional, um caminho extenuante pelo compromisso com a formação contínua para cuidar da vida humana, diante de um sistema capitalista que impõe aos trabalhadores de enfermagem longas horas de trabalho (Mauro, et al., 2019). Assim, os profissionais de enfermagem não conseguem perceber o quão difícil é conciliar o trabalho com a capacitação profissional.

Outro ponto é que o ângulo da visão do gestor de enfermagem mostra as formas com que as equipes foram criadas na pandemia da COVID-19, trazendo realces que podem evidenciar as falhas na formação e/ou capacitação profissional, com evidente aumento dos números de erros no processo assistencial. Todavia, muitos gerentes de enfermagem se focam em muitos aspectos críticos da segurança e qualidade na saúde, mesmos na capacitação profissional (Oliveira, Valente, Messias., \& Souza 2020).

Neste contexto turbulento de contradições, a auditoria interna pode ser uma estratégia transformadora, pois consiste no exame sistemático e independente dos fatos obtidos através de diferentes métodos e técnicas apropriadas, de uma atividade, elemento ou sistema, para verificar se a adequação das ações e seus resultados estão em conformidade aos requisitos preconizados pelas leis e normas vigentes, conforme descrito no planejamento. $\mathrm{Na}$ auditoria, as avaliações são instituídas com o intuito de analisar a estrutura, os processos e os resultados das ações, serviços e sistemas de saúde, com o objetivo de verificar sua adequação aos critérios e parâmetros de eficácia, eficiência e efetividade (Brasil, 1998).

A auditoria interna, mediante suas atividades, deve servir à administração como meio de identificação de todos os procedimentos internos e políticos definidos pela organização (Brasil, 1998). Os serviços de consultoria e assistência são serviços que a organização pode prestar a equipe de auditoria, com o fim de colaborar com eles na implementação de 
adequados mecanismos de controle interno (Chacón, 2014). Portanto, os auditores em enfermagem em auditoria interna na UTI devem ter conhecimento técnico e teórico específico e das diversas áreas relacionadas com as atividades auditadas, o que lhes permitirá comprovar a legitimidade e a legalidade no desempenho dos objetivos dos hospitais.

Na UTI, avaliar as conformidades entre aquilo que as novas equipes interprofissionais podem oferecer e aquilo que a empresa espera deles é bastante difícil, por conta das medidas de isolamento de contato e respiratório. Mas, a auditoria interna da assistência de enfermagem pode ser uma forma de mostrar um caminho possível para a correção das não conformidades no cuidado de enfermagem na COVID-19, visando preparar em longo prazo, a equipe interprofissional de enfermagem para enfrentar e deter, nos próximos meses o avanço da mortalidade da COVID-19.

O estudo tem como relevância mostrar que em situações catastróficas, como na Pandemia da COVID-19, as mudanças de comportamento dos profissionais de saúde podem estar em desconformidade com aquilo que é estabelecido como rotina do trabalho em situações normais. Isso pode se transformar em hábito e modificar-se ao ponto de se transformar em problemas maiores para empresas no contexto futuro.

Tendo em vista que o problema da variabilidade da assistência na saúde não é somente atribuído a enfermagem, em outras áreas econômicas, líderes e empresários estão engajados com a qualidade dos profissionais que estão saindo dos serviços educacionais (Eboli, 2004). Dessa forma, esse estudo se justifica para evidenciar um caminho para outros auditores de enfermagem no afinco desejo do aprimoramento da assistência de enfermagem, mostrando um modelo para executar uma auditoria interna de um hospital, de modo a garantir uma avaliação que modifique a atuação profissional para encontrar a eficácia.

A partir do exposto, descrevem-se as fases da estratificação do problema da qualidade das equipes interprofissional de enfermagem, por meio de uma auditoria interna, para determinar as não conformidades na qualidade das equipes interprofissionais no cuidado ao doente grave com COVID-19, na UTI.

\section{Metodologia}

Trata-se de um relato de experiência da aplicação do modelo de gerenciamento da rotina do trabalho do dia a dia de Campos (2013), na auditoria interna de uma UTI. O gerenciamento da rotina do trabalho do dia a dia é um método de arrumar a casa (empresa) ao tomar providências para que as pessoas, ao exercerem as funções operacionais, sejam as 
melhores do mundo naquilo que fazem (Campos, 2013). A auditoria interna foi solicitada pela gerente de enfermagem do nível tático ao evidenciar que os indicadores da qualidade da assistência de enfermagem haviam aumentado significativamente, após o ingresso das novas equipes interprofissionais para atuar na UTI para casos graves da COVID-19.

Para melhor se adequar ao hospital universitário o modelo foi adaptado em oito etapas: 1) levantamento do problema; 2) descrição do problema; 3) descrição das não conformidades; 4) avaliação do desempenho do profissional de enfermagem; 5) treinamento profissional; 6) avaliação de rendimento na UTI; 7) avaliação de desempenho e, se baixo desempenho, 8) desligamento do projeto. Sendo que este relato de experiência só descreve até a etapa 4.

O cenário foi um hospital universitário localizado na cidade do Rio de Janeiro.

As fases do modelo foram sendo elaboradas no período de março a setembro de 2020. A equipe foi formada por uma auditora, mestre e pesquisadora na área da qualidade, com experiência de 18 anos em doentes considerados de alta complexidade, com a colaboração de quatro gerentes de enfermagem, especialistas em cuidados críticos, que separadamente acumulam cada uma, mais de 10 anos de experiência na docência, assistência e gestão na área de cuidados críticos.

A auditora interna da assistência de enfermagem foi convocada após cinco meses de atuação da nova equipe interprofissional de enfermagem com a finalidade de avaliar as desconformidades em relação à assistência de enfermagem no hospital e estratificar o problema e as metas a serem atingidas até dezembro de 2020.

A pergunta de pesquisa foi: Como vocês avaliam a qualidade da assistência de enfermagem após a chegada de novos profissionais de enfermagem? A escala de resposta levou em consideração cinco constructos: Excelente; muito bom; bom; péssimo e muito ruim categorizados conforme as falas e expressões corporais. A classificação da intensidade da conformidade foi dada pela análise da face, dos gestos e sinais de reprovação. Os demais dados avaliados foram extraídos dos procedimentos operacionais padrões e do checklist do fluxo de trabalho da equipe e registrados em um formulário.

Para análise do problema foi usado o método de análise de Pareto, o ciclo (Plan, Do, Check e Act) PCDA, que é um método de trabalho que leva as pessoas a assumirem responsabilidades de pensar, avaliar e desejar as novas metas. Como define Campos, ele faz com que as pessoas tenham vontade de aprender novos conhecimentos e avaliação do desempenho dos profissionais como determinado. Os dados quantitativos foram analisados por meio de estatística descritiva que forma apenas para expressar simbolicamente as experiencias dos gerentes de enfermagem. 
Research, Society and Development, v. 9, n. 10, e2789108381, 2020

(CC BY 4.0) | ISSN 2525-3409 | DOI: http://dx.doi.org/10.33448/rsd-v9i10.8381

Trata-se de uma demanda interna originada pelo gerente do serviço de enfermagem cirúrgica do hospital. Assim todas as fases da auditoria estavam em acordo com o gestor de enfermagem responsável pelo serviço no nível tático da gestão de enfermagem do hospital e pelo coordenador da assistência de enfermagem da unidade de terapia intensiva. Para manter aspectos éticos, os nomes dos gerentes e do hospital foram mantidos em sigilo.

\section{Resultados}

Logo, nas entrevistas com o coordenador da assistência de enfermagem e uma enfermeiro líder da assistência de enfermagem, ambas revelaram a percepção de piora da qualidade da assistência de enfermagem na UTI por conta do aumento da variabilidade da qualidade da formação dos membros da equipe interprofissional e pelo baixo compromisso em atender às orientações do coordenador da assistência de enfermagem. Algo que foi avaliado por eles na passagem de plantão, nas evoluções de enfermagem e nos aprazamentos dos medicamentos das prescrições médicas, a partir de checklist elaborado com conteúdo extraído dos procedimentos operacionais padrões da instituição.

Em entrevista, ao serem questionadas em relação à experiência acerca da qualidade da assistência de enfermagem, eles responderam que apesar da equipe interprofissional de enfermagem receber informações na passagem de plantão e nas reuniões, a qualidade da assistência de enfermagem ainda continuava muito ruim se comparada ao momento do ingresso dos profissionais na UTI, como mostra figura 1. 
Figura 1. Avaliação da qualidade das equipes interprofissionais de enfermagem após cinco meses de atuação na UTI de casos graves de COVID-19.

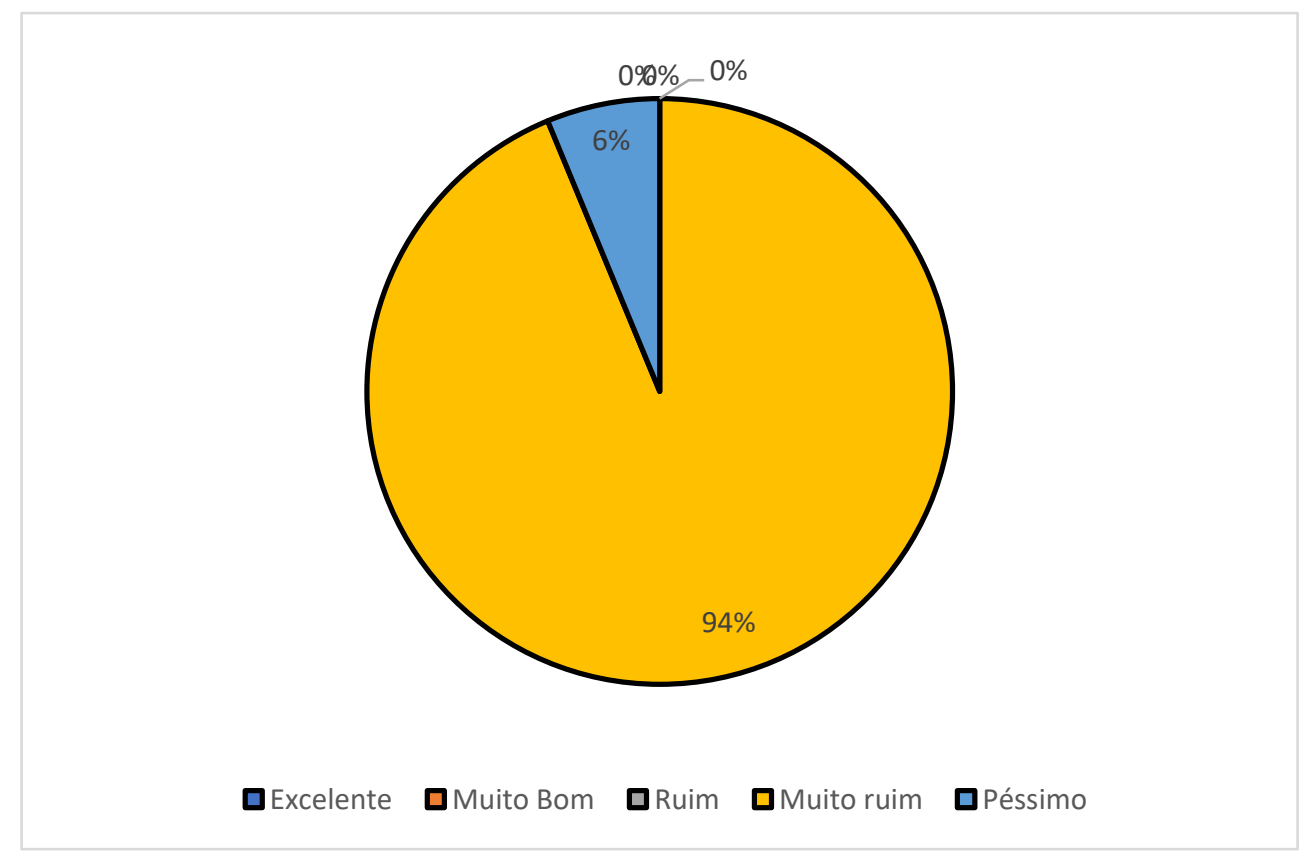

Fonte: autores

A Figura 1 mostra a avaliação das gerentes de enfermagem comparando o desempenho antes e após o ingresso da equipe interprofissional para atuação nos casos graves de COVID19. Através das respostas verbais ao questionamento da auditora, responderam para uma escala de 5 pontos que $94 \%$ da equipe contratada foi considerada muito ruim, ou seja, o nível de conhecimento esperado durante os cinco meses de atuação no hospital, sob supervisão direta, não foi capaz de mudar o comportamento da equipe interprofissional de enfermagem. Os $6 \%$ classificados como péssimos no gráfico, referem-se a alguns membros da equipe, que mesmo após o tempo de atuação na UTI, continuam mantendo comportamentos considerados críticos para segurança do paciente.

Com base nestes dados, foi elaborado um plano de investigação das causas dessas não conformidades que juntos, visam identificar, descrever, implementar, avaliar e intervir nas falhas, diminuindo o impacto no processo de transição de qualificação para o modelo de enfermagem do hospital universitário, melhorando dessa forma o rendimentos da equipe interprofissional (Figura 2). 
Research, Society and Development, v. 9, n. 10, e2789108381, 2020

(CC BY 4.0) | ISSN 2525-3409 | DOI: http://dx.doi.org/10.33448/rsd-v9i10.8381

Figura 2. PCDA do problema da baixa qualidade da assistência de enfermagem aos doentes críticos com COVID-19 grave.

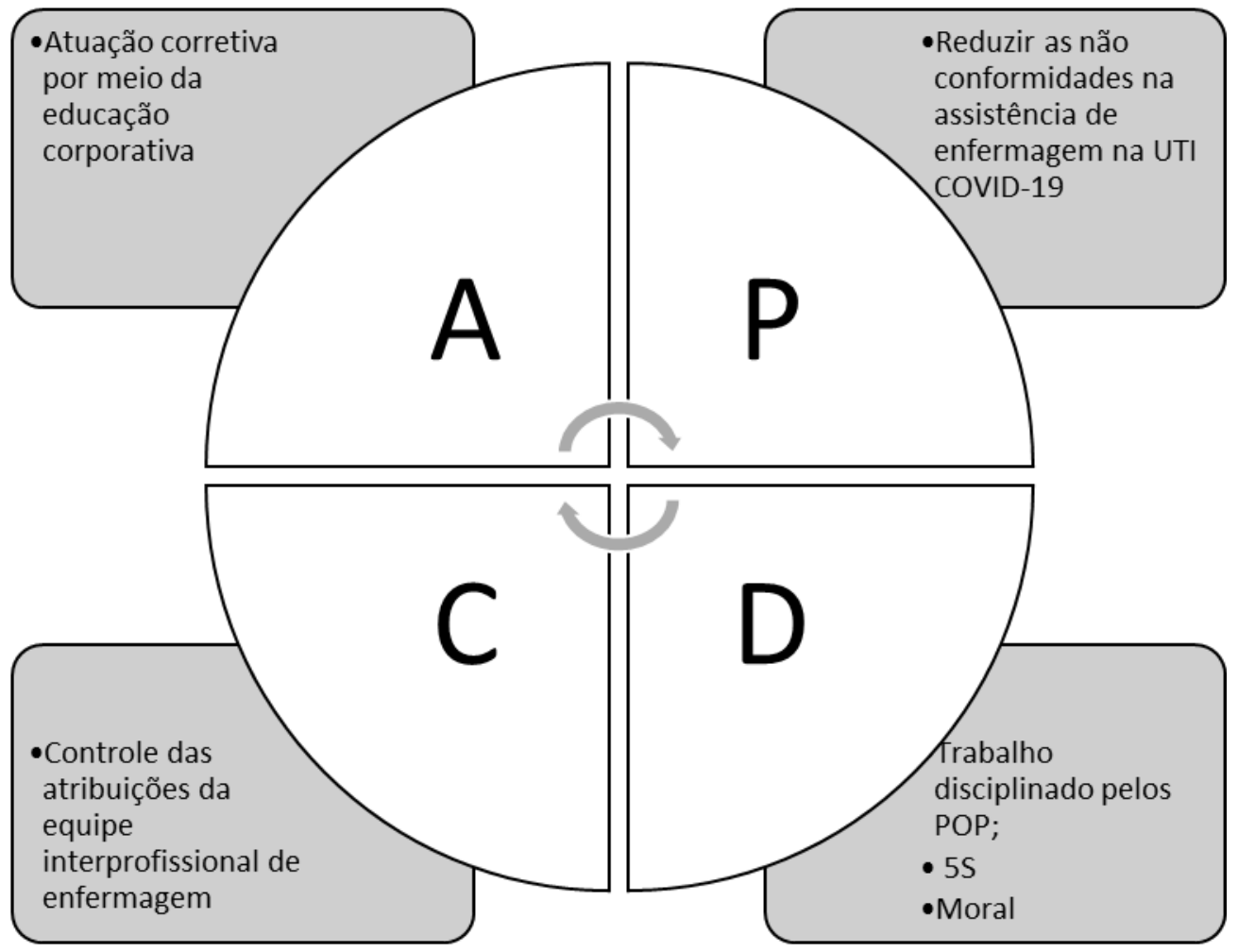

Fonte: Autores.

A Figura 2 mostra o problema relatado: piora da qualidade da assistência de enfermagem antes e depois da transição das equipes de profissionais concursados com os novos profissionais de enfermagem contratados de forma emergencial. A partir da definição do problema, identificou-se que, prioritariamente, em consenso entre o auditor e os gerentes de enfermagem, era preciso resolver o problema de comunicação, como mostram os Quadros 1 e 2 .

Quadro 1. Pareto de comunicação, segundo Campos.

\begin{tabular}{|l|l|}
\hline 1 & $\begin{array}{l}\text { Reduzir as falhas de comunicação entre os profissionais de nível médio e os } \\
\text { enfermeiros; }\end{array}$ \\
\hline 2 & Melhorar as rotulagens das soluções; \\
\hline 3 & $\begin{array}{l}\text { Melhorar a atenção na seleção das vias dos cateteres de infusão para medicamentos } \\
\text { potencialmente perigosos em via única via; }\end{array}$ \\
\hline
\end{tabular}




\begin{tabular}{|c|c|}
\hline 4 & Reduzir as falhas nos registros dos balanços hídricos; \\
\hline 5 & Reduzir as falhas de comunicação nas passagens de plantão; \\
\hline 6 & $\begin{array}{l}\text { Reduzir as falhas de comunicação da equipe interprofissional de enfermagem com a } \\
\text { coordenadora de enfermagem; }\end{array}$ \\
\hline 7 & Melhorar as anotações, registros e avaliações de enfermagem; \\
\hline 8 & Identificação dos acessos venosos profundos e periféricos; \\
\hline 9 & Identificação dos curativos com data e nome do enfermeiro; \\
\hline 10 & $\begin{array}{l}\text { Melhorar a capacidade de distinguir erros no registro de medicamentos nas } \\
\text { prescrições médicas; }\end{array}$ \\
\hline 11 & $\begin{array}{l}\text { Melhorar a capacidade de identificar os medicamentos potencialmente perigosos no } \\
\text { aprazamento das prescrições médicas; }\end{array}$ \\
\hline 12 & $\begin{array}{l}\text { Melhorar os conhecimentos acerca das rotinas de outros serviços de enfermagem na } \\
\text { instituição; }\end{array}$ \\
\hline 13 & Melhorar o perfil de liderança dos enfermeiros; \\
\hline 14 & Melhorar a capacidade de pensamentos críticos em situações de emergência. \\
\hline
\end{tabular}

Fonte: Autoras

Como se pode perceber no quadro 1, o problema é amplo, pois envolve várias áreas de conhecimento em saúde. Percebeu-se também, que o problema tinha relação com a falta de conhecimento do modelo de assistência de enfermagem do hospital. Aprofundado nas análises das entrevistas e dos mapas mentais oferecidos pela coordenadora da assistência de enfermagem, foram elaboradas as metas de redução dos problemas até dezembro (Quadro 2).

Quadro 2. Problemas transformados em metas de correção, segundo Campos.

\begin{tabular}{|l|l|l|}
\hline 1 & $\begin{array}{l}\text { Reduzir as falhas de comunicação entre os profissionais de nível médio e os } \\
\text { enfermeiros; }\end{array}$ & $90 \%$ \\
\hline 2 & Melhorar as rotulagens das infusões; & $100 \%$ \\
\hline 3 & $\begin{array}{l}\text { Melhorar a atenção na seleção das vias dos cateteres de infusão para } \\
\text { medicamentos de infusão em única via; }\end{array}$ & $100 \%$ \\
\hline 4 & Reduzir as falhas nos registros dos balanços hídricos; & $100 \%$ \\
\hline
\end{tabular}


(CC BY 4.0) | ISSN 2525-3409 | DOI: http://dx.doi.org/10.33448/rsd-v9i10.8381

\begin{tabular}{|c|c|c|}
\hline 5 & Reduzir as falhas de comunicação nas passagens de plantão; & $90 \%$ \\
\hline 6 & $\begin{array}{l}\text { Reduzir as falhas de comunicação da equipe operacional com o coordenador } \\
\text { da assistência de enfermagem; }\end{array}$ & $90 \%$ \\
\hline 7 & Melhorar as anotações, registros e avaliações de enfermagem; & $100 \%$ \\
\hline 8 & Identificação dos acessos profundos e periféricos; & $90 \%$ \\
\hline 9 & Identificação dos curativos com data e nome do enfermeiro; & $90 \%$ \\
\hline 10 & Melhorar a capacidade de distinguir erros nas prescrições médicas; & $90 \%$ \\
\hline 11 & $\begin{array}{l}\text { Melhorar a capacidade de identificar os medicamentos potencialmente } \\
\text { perigosos no aprazamento das prescrições médicas; }\end{array}$ & $90 \%$ \\
\hline 12 & $\begin{array}{l}\text { Melhorar os conhecimentos acerca das rotinas da comissão prevenção de } \\
\text { lesões de pele e curativos da instituição. }\end{array}$ & $90 \%$ \\
\hline
\end{tabular}

Fonte: Autoras.

\section{Discussão}

A qualidade é um conceito muito usado na enfermagem e talvez por isso, as enfermeiras responsáveis pela assistência de enfermagem se preocuparam tanto com a queda dos indicadores de qualidade (Ferreira, et al., 2020). A necessidade de instituir uma auditoria interna surgiu a partir da análise destes indicadores que revelaram o aumento dos eventos adversos associados à assistência de enfermagem. A gerente do serviço de enfermagem em cirurgia responsável pelo gerenciamento tático da UTI tem o controle rigoroso da qualidade, ao ponto de ela conseguir identificar precocemente anomalias advindas do comportamento profissional e da falta de capacitação profissional e pedir uma auditoria interna.

A ação só foi possível porque a instituição auditada é uma das poucas no Brasil que tem uma alta gestão de enfermagem e por isso ela possui uma diretriz formulada para o enfrentamento da pandemia da COVID. Campos (2013) aponta que a sua experiência nas empresas tem mostrado, de forma muito clara, que o alcance de metas pode ser garantido desde que as lideranças estejam dispostas a seguir fielmente um determinado ritual, que engloba agenda anual de reuniões de acompanhamento e um bom sistema de informações. Com isso em mente, a primeira fase na auditoria interna foi pensada para levantar o problema e estratificá-lo em partes menores com bases nestas diretrizes (Campos, 2013). 
Research, Society and Development, v. 9, n. 10, e2789108381, 2020

(CC BY 4.0) | ISSN 2525-3409 | DOI: http://dx.doi.org/10.33448/rsd-v9i10.8381

Dessa forma, a escolha do modelo de gestão da qualidade total só foi elencada após as entrevistas com os gerentes de enfermagem, a visitação aos cenários e a análise dos registros de enfermagem. Depois disso, os dados foram confrontados com as diretrizes do diretor de enfermagem, por isso o auditor da qualidade só conseguiu pensar no modelo de gerenciamento da rotina do trabalho do dia a dia após ter levantado as anomalias encontradas na UTI.

Qualitativamente, a entrevista com os gerentes de enfermagem teve a finalidade de analisar o corpo como símbolo, pois ele atua na direção de um sentido. Sentido que se desvela, ou seja, a consciência de ser esse corpo que fala, que pensa, que sente, que age (Mendonça, 2004). Durante a entrevista, o coordenador de enfermagem chorou pela experiência de desamparo com a nova equipe interprofissional. Era um corpo encurvado e entristecido que denunciava o universo consciente e inconsciente do desassossego do afastamento do seu grupo anterior, que foi a referência para sua capacitação profissional, após concurso público. Assim, percebeu-se que o desassossego do coordenador de enfermagem estava associado ao tempo que ela trabalha junto de sua equipe interprofissional antiga, havia um profundo pesar com a ruptura do objetivo compartilhado por eles.

A observação e a análise simbólica corporal foram imprescindíveis para a categorização do grau de insatisfação do coordenador da assistência de enfermagem e do gerente tático de enfermagem com a qualidade da equipe interprofissional de enfermagem que ingressou através de contrato terceirizado, dando intensidade emocional à avaliação. Eles estavam temerosos pelos doentes e de como a equipe interprofissional de enfermagem se comportava e com a falta de empenho em buscar por novos conhecimentos.

Em relação às equipes que trabalham muito tempo juntas, geralmente seus membros se conhecem bem e conseguem identificar os pontos fortes, os valores e as habilidades de cada parceiro. Eles também conhecem suas vulnerabilidades e a forma como enfrentam situações difíceis, o que traz vantagens às equipes reais, algo que a coordenadora de enfermagem perdeu abruptamente (Mosser, \& Begun., 2015).

O plano de correção da qualidade por meio da Educação Corporativa (EC) tem o propósito de que cada empresa aprenda a trabalhar com novos processos ao criar novas soluções, a fim de que a mesma vislumbre a importância da aprendizagem permanente vinculada à meta empresarial (Eboli, 2004). Ela também defende que a missão da EC consiste em formar e desenvolver os talentos na gestão dos negócios e assim promover a gestão do conhecimento organizacional. Assim, acreditamos que a EC seja uma importante ferramenta 
para aprendizagem das diretrizes organizacionais de enfermagem pelas suas equipes interprofissionais, buscando o desenvolvimento de talentos que agregam valor ao hospital.

Pois, esse modelo de gerenciamento tem como base as diretrizes da alta gestão, focando-se em arrumar a casa, concentrando as mentes e as ações dos gerentes em cinco itens: a) escolha a área de sua empresa que não vai bem. Inicie arrumando pelo pior local; b) defina os problemas dessa área, estando ciente que podem surgir problemas localizados num mesmo setor ou problema interfuncional de caráter amplo; c) os problemas interfuncionais devem ser desdobrados em problemas localizados; d) para cada problema localizado defina um item de controle; e) planeje atingir as metas para seis meses; e f) foque apenas nas estratégias em atingir as metas (Campos, 2013). A partir desse processo seguiu-se os seguintes passos: a) o problema pior no hospital era a equipe interprofissional de enfermagem da UTI, criada para tratar as formas graves de COVID-19; b) o problema foi identificado como queda da qualidade da assistência de enfermagem; c) o problema interfuncional foi a educação permanente do hospital que não conhecia as estratégias de educação corporativa; d) o item de controle foi o checklist da passagem de plantão da coordenadora da assistência de enfermagem; e) por conta da gravidade do caso a avaliação da meta foi instituída para três meses; e ) o foco foi mudar a estratégia de supervisão direta para capacitação profissional com temas relacionados às metas de segurança dos pacientes.

Um auditor interno é um funcionário da empresa que por ele será auditada, e por isso, possui menor grau de independência se comparado a um auditor externo. No entanto, exerce com mais profundidade auditoria no nível operacional, seja na área financeira, de qualidade, de processos, de produção e outras (Brasil, 1998). Desse modo, este relato de experiência mostra o que muitos autores na área destacam, a auditoria é considerada uma das mais importantes ferramentas para delinear os caminhos na busca pela qualidade. Pensando nisso, os gerentes de enfermagem na presença de uma anomalia recorreram a um membro da sua equipe especialista em gestão da qualidade total para dar caminhos as suas inquietações. Esse dado corrobora com um estudo que demonstra que os enfermeiros de um hospital universitário advogam que é importante ter um enfermeiro chefe, formado em gestão na UTI (Macedo, Padilha., \& Püschel., 2019).

O coordenador assistência de enfermagem ofereceu os Procedimentos Operacionais Padrão (POP) da assistência de enfermagem e, após a análise dos mesmos, foram classificados em não conformidade, pois não foram elaborados seguindo a recomendação da Agência Nacional de Vigilância Sanitária (ANVISA). Os mesmos continham descrições extensas com processos, conceitos, definições, intervenções e diversos processos de 
enfermagem. Destaca-se que a constituição de um POP é um conjunto de listas contendo: materiais necessários; passos críticos; manuseio do material; resultados esperados; e ações corretivas (Campos, 2013). Ele elucida que essa ferramenta melhora as atividades dos profissionais da ponta, servindo como uma forma de treinar o pessoal.

O conhecimento das diretrizes da direção da enfermagem do hospital auditado, bem como a análise dos POP são imprescindíveis nas avaliações. As informações levantadas com as avaliações servem de conteúdo para as aulas corretivas, onde muitas vezes os auditores de enfermagem recomendam o abandono de modelos que foram caros e penosos de serem instituídos, mas que desequilibram as certezas na atuação profissional e trazem ansiedade diante do novo. Talvez, por conta disso, é incomum auditorias de enfermagem que combinam avaliações e ações corretivas por meio de educação corporativa.

Antes mesmo da Pandemia da COVID-19, as estratégias de capacitação profissional já se mostravam deficientes no hospital de ensino e isso piorou ainda mais com a saída das equipes antigas e a entrada das novas equipes no cenário (Oliveira, Valente, Messias., \& Souza, 2020). Talvez, o uso ininterrupto do EPI nos ambientes hospitalares tornou mais complexo e incerto ao limitar, de certa forma, a cooperação entre as equipes interprofissionais de gerações diferentes para cuidar de pessoas. Nesta situação, parece que as máscaras e os capotes de proteção também se tornaram mais uma barreira na comunicação das experiências, na assistência de enfermagem ao doente crítico com COVID-19. E, aquilo que é essencial para evitar a disseminação do vírus se tornou algo que pode ter minimizado os efeitos da supervisão direta de enfermagem. Destaca-se dessa forma, que não se tem conhecimento como os EPI interferem na comunicação das equipes interprofissionais e intergeracionais.

Como em todas as atividades econômicas, as de enfermagem estão relacionadas à produção de produtos ou à prestação de serviços (Balbino, et al., 2020). Sendo planejadas, coordenadas, dirigidas, executadas e controladas pelos enfermeiros. A preocupação com esses produtos e serviços, processos, funções, doentes, fornecedores e custos está diretamente relacionada com o desempenho da qualidade das equipes interprofissionais de enfermagem.

Esse estudo tem como limitação as experiências vivenciadas pelas gerentes de enfermagem do hospital universitário auditado, podendo ser diferentes de outros hospitais em decorrência da sua estrutura organizacional, que tem um diretor de enfermagem no mesmo nível do diretor médico, além de enfermeiros gerentes comprometidos com a segurança e qualidade da assistência de enfermagem. Mesmo com essa característica diferenciada, os resultados podem não ser atingidos por conta de fatores organizacionais, comportamentais, culturais, econômicos e operacionais. 


\section{Considerações Finais}

A Pandemia da COVID-19 colocou os gestão de enfermagem diante de uma nova situação em hospitais públicos, a entrada de novos membros na equipe interprofissional de enfermagem de forma não planejada e com baixa capacitação em cuidados críticos. Essas equipes interprofissionais apresentam baixo desempenho com a supervisão direta de enfermagem mesmo após cinco meses de atuação na UTI, talvez porque acumulem costumes de outros hospitais ou até mesmo porque nunca haviam trabalhado em hospitais. Desse modo, o problema é multifatorial e resvalou de forma acentuada nos gerentes de enfermagem concursados do hospital acostumados com elevado nível de capacitação de seus profissionais de enfermagem.

O compromisso com o controle de qualidade da assistência de enfermagem pela gerência de enfermagem do hospital foi essencial para, diante da mudança abrupta da equipe interprofissional de enfermagem, sinalizar que o aumento da mortalidade na unidade, ao notar que os parâmetros estavam divergindo das medidas padrões anteriores, foi o sinalizador da anomalia. Constatou-se também que as anomalias tinham de alguma forma uma relação (direta ou indireta) com a ausência de intervenções de enfermagem levantadas no checklist de qualidade da passagem de plantão, tais como: cabeceira do leito fora do parâmetro de 30 a 45 graus, medicamentos incompatíveis fluindo numa mesma via do cateter venoso central, falta de higiene oral três vezes por dia e outras intervenções de enfermagem consideradas essenciais pelas metas de segurança do paciente e pelos procedimentos operacionais padrões da instituição.

Apesar disso tudo, a supervisão direta da assistência não se mostrou uma ferramenta efetiva para mudar o comportamento das equipes com a assistência de enfermagem. Acreditase que esse fato se deve a urgência da admissão de novos profissionais de enfermagem pelo hospital. Os baixos salários, o uso ininterrupto de EPI e o acúmulos de vários empregos e a péssima capacitação nos cuidados críticos foram os motivos das anomalias encontradas na auditoria interna e a dificuldade de se instituir a supervisão direta no controle da qualidade da assistência de enfermagem.

Os gerentes de enfermagem recorreram a um enfermeiro altamente capacitado em um modelo gestão da qualidade total que atuava como líder no hospital. Mesmo ele sendo um líder, trata-se de um enfermeiro de nível elevado de performance na gestão da qualidade, algo 
que deve ser fomentado em outros hospitais como forma de ter um enfermeiro capaz de identificar, avaliar e prescrever ações para resolver as anomalias da qualidade sensíveis a assistência de enfermagem.

A análise simbólica corporal evidenciou o desamparo sentido pelo coordenador de enfermagem pelo afastamento da sua equipe interprofissional e a falta de apoio da educação permanente do hospital às necessidades de capacitação profissional, levantadas pelo coordenador de enfermagem, teve um grande impacto na sua saúde mental de ambos. Neste caso, pensar em bônus como uma folga no meio da semana pode ser uma estratégia para minimizar o sofrimento pela ruptura de sua equipe interprofissional de enfermagem antiga e o sentimento de desamparo.

Por fim, a partir da metodologia do gerenciamento da rotina do trabalho do dia a dia, foi possível estratificar o problema, traçar as metas de redução das falhas até dezembro, pensar na educação corporativa como uma estratégia de capacitação profissional para as correções das falhas e mostrar que a troca de equipes interprofissionais de enfermagem inteiras num mesmo momento é uma anomalia grave para a segurança do paciente. Desse modo, recomenda-se que o gestor de enfermagem elabore as suas diretrizes para que os gerentes da qualidade consigam usar o método de arrumar a casa de Campos.

\section{Referências}

Brasil. (1998). Manual de normas de auditoria. Brasília: Ministério da Saúde.

Campos, V. F. (2019). Gerenciamento da Rotina do Trabalho do Dia-a-Dia; $9^{a}$ ed. Rio de Janeiro: Falconi.

Chacón, J. I. A. (2015). Auditoria governamental e tipos de serviços de auditoria prestados pela equipe de auditoria governamental. $R C \& C .7$ (2), 127-143. doi: 10.5380/rcc.v7i2.38165.

Eboli, M. (2004). Educação Corporativa no Brasil: mitos e verdades. (3 ${ }^{\text {a }}$ ed.) São Paulo: Gente.

Ferreira, A. O. M., Silvino, Z. R., Balbino, C. M., Izu, M., Souza, C. J., \& Matias, D. O. (2020). Modelo multicritério para controle de qualidade de fraldas geriátricas de uso 
hospitalar. Research, Society and Development, 9 (7), e753974835. doi: 10.33448/rsdv9i7.4835.

Figueiredo, T. O. (2020). Estratégias para operacionalização e sistematização da assistência de enfermagem em cenário hospitalar no enfretamento à pandemia COVID-19, Saúde Coletiva. 54(10).

Macedo, A. P. M. C., Padilha, K.G., \& Püschel, V.A.A. (2019). Práticas profissionais de educação/formação dos enfermeiros em uma unidade de cuidados intensivos. Rev Bras Enferm 72(2), 321-328. doi: 10.1590/0034-7167-2017-0793.

Mauro, M. Y. C., Rabelo, A.M. S., Ferreira, A. O. M., Sper, N. P T., \& Santos, M. I. S. (2019). Night work and self-perceived body weight changes among nursing professionals. Revista Enfermagem UERJ, 27, e31273. doi:10.12957/reuerj.2019.31273.

Mendonça, J. G. R. (2004). O corpo e sua dimensão simbólica. Presença, 29(3), 1-13.

Mosser, G.; \& Begun, J. W. Compreendendo o trabalho e equipe de saúde. Porto Alegre: AMGH, 2015.

Oliveira, P. V. N, Valente, G. S. C., Messias, C. M., \& de Souza, C. J. (2020). A formação permanente do enfermeiro e sua inserção na terapia intensiva do hospital universitário. Research, Society and Development, 9(7), e651974533. doi: 10.33448/rsd-v9i7.4533.

Trovó, S. A., Cucolo, D. F., Perroca, M. G., Trovó, S. A., Cucolo, D. F., \& Perroca, M. G. (2020). Time and quality of admissions: nursing workload. Rev Bras Enferm, 73(5). doi: 10.1590/0034-7167-2019-0267.

\section{Porcentagem de contribuição de cada autor no manuscrito}

Alexandra de Oliveira Matias Ferreira - 90\%

Zenith Rosa Silvino - 10\% 